accidentelles Geräusch auszuschliessen, da solche in diastolischer Form fast niemals vorkommen. Endlich blieb als die wahrscheinlichste Annahme die übrig, dass das Geräusch im Aneurysma selbst erzeugt sei, wie es gerade bei Bauchaortaaneurysmen schon frïher beobachtet war. Bestimmter Aufschluss darüber war natürliclı luu durch die Obduktion zu erwarten.

In einer Sitzung des Danziger ärztlichen Vereins, in der der Patient vorgestellt wurde, demonstrirte Geh. Rath $\mathrm{S}$ che ele in selir instruktiver

V. Aus der inneren Station des Diakonissenkrankenhauses in Danzig.

(Oberarzt: Geh. San.-Rath Dr. Scheele.)

\section{Ein bemerkenswerther Fall von Aneurysma dissecans aortae abdominalis.}

\section{Von Dr. Ernst Fast in Praust bei Danzig.}

Angeregt durch die Veröffentlichung eines Falles von Aneurysma der Aorta abdominalis durch Geh. Rath v. Leyden in No. 23 der Deutschen medizinischen Wochenschrift, theile ich nachstehend einen weiteren Fall dieser ausserordentlich seltenen Erkrankung mit, der bereits im Jahre 1897 von Geh. Rath Scheele und mir im Diakonissenkrankenhause zu Danzig beobachtet wurde. Die Publikation desselben lag damals schon in meiner Absicht, unterblieb jedoch aus äusseren Gründen. Der Fall bietet gerade in Parallelstellung zu dem Leyden'schen, dann aber auch wegen verschiedener Besonderheiten und des Sektionsergebnisses soviel Interessantes, dass seine Wiedergabe auch jetzt noch von Werth erscheint

Krankengeschichte: Der 42 Jahre alte Kohlenarbeiter L. M. aus Danzig wurde am 28. März 1897 auf die innere Station des Diakonissenkrankenhauses aufgenommen. Bei seiner Beschäftigung als Kohlenarbeiter hat er stets sehr schwer zu arbeiten gehabt, auch giebt er Abusus potus zu. Seit vier Jahren schon klagte er über Rückenund Kreuzschmerzen. Dieselben zogen sich allmählich mehr nach der linken Seite, Hiifte und vorderem Oberschenkel. Vor einem halben Jahre erst holte er ärztlichen Rath ein und wurde für leberkrank, dann für rheumatisch leidend erklärt. Im Winter sollen die Schmerzen stets heftiger aufgetreten sein als im Sommer. Seit drei Wochen ist Patient wegen exzessiver Zunahme der Beschwerden arbeitsunfähig. Eine frühere spezifische Infektion ist nicht eruirbar.

Status: Bei der Betrachtung des entblössten Rumpfes fällt ausser sehr lebhafter Erschütterung der Herzgegend eine Pulsation unterhalb des rechten Rippenrandes auf. Deutlicher noch fühlt man dieselbe. Beim palpatorischen Vergleich des Herzstosses und dieser Pulsation zeigt sich, dass beide durch ein deutlich wahrnehmbares Intervall zeitlich von einander getrennt sind. Der Herzstoss geht der Hebung des Rippenrandes rechts um einen Moment voraus. Die Palpation der grossen Gefässstämme, der Carotiden, der Crural- und der Radialarterien ergiebt nichts Charakteristisches. Im Jugulum ist wohl eine leichte Pulsation zu fühlen, die Carotiden und die Radialarterien zeigen aber nur eine vermehrte Spannung, kein Schwirren und Hüpfen. Die Cruralpulse sind quoad tempus et volumen, sowie in der Wellenform nicht merklich unterschieden.

Perkutorisch lässt sich nur eine Dilatation des Herzens nach links constatiren. Auf dem Manubrium sterni keine deutliche Dämpfung.

Auskultatorisch hört man an der Herzspitze (6. Intercostalraum), $1 \mathrm{~cm}$ ausserhalb der Papillarlinie einen dumpfen ersten und einen reinen lauten zweiten Ton. Mitten auf dem Sternum, dicht oberhalb des Processus ensiformis hört man ein fast metallisch klingendes, ex quisit diasto lis ch esGeräus ch, das sich nach dem Ostium aorticum und pulmonale hin verfolgen lässt, dort jedoch in seiner Intensität wesentlich schwächer ist. Ueber dem pulsirenden Tumor ein dumpfes systolisches Geräusch. In beiden Carotiden deutlicher zweiter Ton ohne Geräusch. Druck auf beide Arteriae crurales, die beider seits gleich stark gefüllt sind, lässt das diastolische Geräuschlauter werden.

Auf der Haut des Thorax sind einige erweiterte geschlängelte Venen sichtbar.

Anzeichen überstandener Lues nicht nachweislich

Urin: Spezifisches Gewicht 1012, sauer, schwach Spuren von Albumen. Mikroskopisch nichts Pathologisches.

Auf den physikalischen Befund hin wurde die Diagnose auf ein Aneurysma der Abdominalaorta gestellt. Schon die ausgesprochenen, von einander zeitlich zu sondernden Pulsationen, die Erscheinung „zweier Herzen in einer Brust" (Stokes), war dafür ganz bezeichnend. Es wurde nur die Möglichkeit eines Aneurysma der Arteria coeliaca offen gelassen. Dies geschah auf Grund des Befundes an den Cruralarterien, die weder eine zeitliche Verspätung noch eine Veränderung im Volumen und Wellenform erkennen liessen. Die physikalische Erscheinung des diastolischen Geräusches über dem Processus xyphoideus liess verschiedene Deutungen zu. Die nächstliegende einer gleichzeitig bestehenden Aortenklappeninsufficienz musste in $Z_{\text {weifel }}$ gezogen werden, da die peripheren Arterien die dafür charakteristischen Zeichen nicht finden liessen. Ebenso war ein sogenanntes Weise die zeitliche Differenz der Pulsationen am Cor und Aneurysma so, dass er allf die Centren der pulsirenden Particen zwei an Pappreiterchen angeklebte Kehlkopfspiegelchen aufsetzte. Das von diesen reflektirte Licht einer Lichtquelle wurde auf einem weissen Leinwandschirm aufgefangen. So liess sich die verspätete Aktion des Arterientumors ein wandsfrei veranschaulichen.

Der Fall verlief dann in der Weise weiter, dass trotz Eisapplikation, Darreichung von Digitalis und Jodkali der Tumor sicl ver grösserte und immer deutlicher in die Erscheinung trat. Fieber wurde nur einmal interkurrent beobachtet. Die wahnsinnigen Schmerzen des Patienten wurden durch steigende Morphingaben gemildert. Fünf Wochen ante exitum trat während einer Nacht Oedem des linken Unterarm und Hand- und Fussriickens ein, das in dell nüchsten Tagen auch den rechten Unterschenkel und Fuss ergriff, verbunden mit zeitweiser Urirverminderung. Nach drei Wochen verschwanden die Oedeme spontan. Sechs Tage vor dem Ende stellten sich abendliche Temperaturerhöhungen eiı. Am 1. Juli erfolgte unter den Bilde progredienten Kräfteverfalls der Exitus.

Sektions befund: Sehr magere männliche Leiche, äusserlich, abgesehen von gleichmässiger Auftreibung des Abdomens, nichts Auffalliges.

Cor bedeutend vergrössert. Im Herzbeutel circa ein Esslöffel Flüssigkeit. In den Stümpfen der grossen Arterien stecken grosse, bis in die Coronargefässe hinein sicl verzweicende, zum Theil organisirte, zum Theil speckartige Thromben. Nach Entfernung der Gerinnsel wird die Wasserprobe angestellt. Sie ergiebt exquisite Schlussfähigkeit der Aortenklappen. Rechter Ventrikel dilatirt, Muskelwandung sehr dünn, linker Ventrikel dilatirt und hypertrophisch. Die aufsteigende Aorta und der Aortenbogen cylindrisch erweitert. Ausgedehnte Verfettung und Verkalkung der Intima der Aorta. Die Klappensegel frei.

Linke Lunge ausgedehnt adhärent, kaum zu lösende Verwachsungen in der Spitze.

Rechte Lunge ebenfalls zum Theil verwaclısen, lässt sich jedoch leichter auslösen.

In beiden Spitzen käsige Heerde.

Abdomen enthält circa $1 / 2$ Liter blutiger Flüssigkeit. Beim Zurückschlagen der Därme nach rechts fällt in der linken Bauchhälfte ein kindskopfgrosser gestreckter Tumor auf, der eine blind endigende, faustgrosse Geschwulst bis ins kleine Becken hineinsendet. Diese Geschwulstmasse besteht aus mehr oder weniger coagulirten, zum Theil fibrinösen Blutmassen (Hämatoma retroperitoneale).

Leber mässig vergrössert, hart, mit höckeriger Oberfläclıe (Cirrhose). Keine narbigen Einziehungen, bezw. gummöse Stellen.

Nach Entfernung der Därme, des Magens, der rechten Niere, der Milz und des Diaphragma liegt ein Tumor von der Grösse zweier Fäuste vor, der sich dem vorhergenannten links gelegenen Blutklumpen anlagert. Aus der vorderen Wand dieses 'Tumors entspringt ein kleinfingerdickes arterielles Gefäss, das sich deutlich nach dem Mesenterium hin verfolgen lässt (erweiterte Arteria mesenterica). Es wird nun die Brust- und der unterste Theil der Bauchaorta ganz freigelegt. Die erstere zeigt in der Gegend des Hiatus aorticus eine allmällich zunehmende Erweiterung. Nachdem die Schenkel des Hiatus abpräparirt sind, sieht man unter ihnen sich birnförmig vergrössernd eine von der Adventitia des Gefässrohrs bedeckte aneurysmatische Erweiterung der Aorta. Das ganze Gefässrohr der Brustaorta wird aufgeschnitten bis zu der beginnenden Erweiterung hin, und der zufühlende Finger gelangt in den mit festen Thromben gefiillten aneurysmatischen Sack. Die Weite des Gefässrohrs in der Gegend des Hiatıs aorticus ist geringer als die oberhalb und unterhalb dieser Stelle gelegene Aortenpartie. Von des Theilungsstelle der beiden Arteriae iliacae beginnend, wird jetzt die Aorta nach oben hin verfolgt. Sie geht in der Höhe des zweiten Lendenwirbels in jenen aneurysmatischen Sack über. Spaltung des ganzen Gefässtraktus durch Scheerenschnitt. Man sieht jetzt in eine etwa gänseeigrosse Höhle, deren Wand nach vorn kugelig vorgetrieben und stark verdickt ist. Zwwischen dem innern und dem äussern Blatt liegt eine dicke Schicht ziemlich consistenten organisirten Blutgerinisels, Die hintere Wand der ziemlich unregelmässig gestalteten Höhle ist dünn und zeigt einen queren, etwas nach links von der Wirbelsänle befindlichen, circa $2 \mathrm{~cm}$ langen Riss mit unregelmässigen Rändern, durch den man auf den Bluterguss kommt. Das Lumen der Aorta ist oberhalb des Tumors gut für zwei, unterhalb eben für einen Finger durchgängig.

Nach sorgfältiger Ablösung der ganzen Geschwulstmasse und der Aorta finden sich der 12. Brust- und 1. und 2. Lendenwirbelkörper tief usurirt. Die Intervertebralscheiben sind fast vollständig erhalten und springen vor. Der Wirbelkanal ist nicht eröffnet.

An den übrigen Organen der Bauchhöhle findet sich sonst nichts 
Bemerkenswerthes, nur in der rechten Vena iliaca zeigen sich mehrere regenwurmartige, zum Theil an der Gefässwand adhärente organisirte Thromben.

Anatomische Diagnose: Dilatatio cordis, Hypertrophia ventriculi sinistri, Dilatatio aortae cylindriformis. Atheromasia aortae multiplex. Aneurysma dissecans aortae abdominalis. Haematoma retroperitoneale permagnum. Hepatitis interstitialis chronica.

Epikrise: Aus dem Sektionsbefunde ergiebt sich die Bestätigung der intra vitam gestellten Diagnose auf ein Aneurysma der Abdominalaorta. Das retroperitoneale Hämatom war zu Lebzeiten des Patienten nicht diagnostizirt worden, weil, wie aus der starken Gerjnnselbildung in den Blutklumpen zu ersehen war, die innere Verblutung äusserst langsam erfolgte, also keine Erscheinungen akuter Anämie bewirkte und die Schmerzen des Patienten, bezw. eventuelles Erbrechen durch Peritonealreizung, die sonst wohl darauf hingedeutet hätten, mit Morphin unterdrückt waren. Aus dem Symptomencomplex, der während des Lebens beobachtet war, verdient eine ganz besondere Berücksichtigung das diastolische Geräusch, welches am deutlichsten dicht oberhalb des Processus xiphoideus mit einer gleichmässigen Constanz gehört wurde. Es lag, wie erwähnt, nahe, dieses diastolische Geräusch, das sich fast bis zum zweiten Intercostalraum links, wenn auch in abgeschwächter Form verfolgen liess, auf eine gleichzeitig bestehende Aorteninsufficienz zu beziehen, und das um so mehr, als sich eine einwandsfreie Hypertrophie und Dilatation des linken Ventrikels nachweisen liess. Allein damit stimmte, wie bereits bemerkt, nicht überein die Beschaffenheit der peripheren Arterien. Denn wenn auch die Carotiden prall gefüllt waren und eine gewisse Spannung zeigten und der Aortenbogen sich im Jugulum palpiren liess, so fehlte doch das charakteristische Hüpfen und Schwirren der Carotiden, es fehlte der Pulsus celer an der Radialis. Dieses Verhalten del peripheren Arterien blieb während der ganzen Zeit der Beobachtung constant das gleiche. Auch auskultatorisch trat keine Aenderung ein, namentlich zeigte sich nie, dass der zweite Ton an den Carotiden ausfiel, ein Symptom, das bei Aorteninsufficienz, wenn auch nicht immer, so doch ziemlich regelmässig zu beobachten ist, ebensowenig war das diastolische Geräusch in den Carotiden wahrzunehmen, was bei diesem Klappenfehler gelegentlich vorkommt. Es wurde bei der Bes.prechung der Diagnose demgemäss schon hervorgehoben, dass der Ursprung dieses diastolischen Geräusches in das Aneurysma selbst verlegt werden könnte, und diese Auffassung bestätigte der Sektionsbefund. Es zeigte sich, dass die Aortenklappen bei der Wasserprobe vollständig schlossen. Es zeigte sich ferner an den Semilunarklappen weder ein rétrécissement, noch auch eine Andeutung einer relativen Insufficienz. Wie ist nun die Entstehung des diastolischen Geräusches zu erklären? Darüber gab der Befund an der Eingangspforte des Aneurysma im Hiatus aorticus hinreichenden Aufschluss. Die an jener Stelle befindliche Verengerung des Arterienrohres musste während der Diastole ein geringes Zurückströmen des Blutes aus dem erweiterten Aortensack in die vor der verengten Stelle befindliche erweiterte Brustaorta zu Stande kommen lassen. Somit entstand eine gewisse diastolische Wirbelbildung und dadurch das diastolische Geräusch. In den aneurysmatischen Sack selbst, wie es in seltenen Fällen früher schon beobachtet worden ist, konnte im vorliegenden Falle die Entstehung des diastolischen Geräusches wohl kaum verlegt werden, da nach dem anatomischen Befund die Höhle des Aneurysma - etwa von Birnengrösse - um so weniger gross genug war, als sie auch noch mit zum Theil organisirten Thromben sich angefüllt zeigte.

Das über dem Aortenaneurysma selbst unterhalb des rechten Rippenrandes gehörte systolische Geräusch bedarf bei der Menge von höckrigen fibrinösen Niederschlägen an der Aneurysmawand keiner besonderen physikalisehen Erklärung.

Weiterhin ist epikritisch hervorzuheben das Verhalten der peripheren Arterien unterhalb des Aneurysma. Es fand sich quoad volumen, tempus et amplitudinem keine nennenswerthe Veränderung. Diese Erscheinung veranlasste dazu, bei der Stellung der Diagnose die Möglichkeit eines Aneurysma arteriae coeliacae ins Auge zu fassen, denn es musste auffallen, dass bei einer eingeschalteten Erweiterung des Bauchaortarohres, die für das Gefühl eine ziemlich beträchtliche zu sein schien, die Cruralarterien keine bezüglichen physikalischen Abweichungen erkennen liessen. Die Sektion liess jedoch eine Erkrankung der Arteria coeliaca nicht erkennen. Zur Erklärung bleibt nur der
Umstand übrig, dass der aneurysmatische Sack der Bauchaorta so gleichmässig mit Gerinnseln ausgefüllt war, dass ein erweitertes Lumen eigentlich fehlte und der Durchmesser der Aneurysmahöhlung nicht wesentlich weiter war als der Querschnitt des Gefässes selbst. Das ist besonders lehrreich, weil der Fall beweist, dass gelegentlich die am häufigsten und gewissermaassen pathognomonischen Zeichen ausfallen können.

Ferner bedarf wohl auch noch einer epikritischen Beleuchtung der Umstand, dass bei Compression der Cruralarterien sich durch die Auskultation eine Intensitätsänderung, eine Verstärkung des diastolischen Geräusches constatiren liess. Dieselbe ist wohl kaum anders zu erklären, als dass durch den plötzlichen Verschluss der Cruralarterien der Blutdruck innerhalb der Aorta einen gewissen Zuwachs erhielt.

Endlich erübrigt noch die Frage: Wann ist die Ruptur des Sackes eingetreten?

Wie aus der Krankengeschichte hervorgeht, stellten sich in Folge übermässig gewordenen Druckes seitens des wachsenden Aneurysma fünf Wochen ante exitum Oedeme ein, die allmählich zunahmen. Das Erscheinen des Anasarka ist offenbar auf eine Behinderung der Cirkulation in der Vena cava inferior, auf eine seitliche Compression derselben zurückzuführen. Auffallend war, dass dieses Symptom nicht bis zum Tode des Patienten fortbestand. Diese Erscheinung findet ihre Erklärung in der ganz allmählich eingetretenen Ruptur der Aneurysmawand. Damit musste natürlich der Druck in dem Blutsack sinken und die Venenbahn wieder frei werden. Man muss also annehmen, dass mindestens 14 Tage ante exitum, als die Oedeme ganz verschwunden waren, die Ruptur des Aneurysma bereits erfolgt war.

Ich möchte diesen casuistischen Beitrag nicht abschliessen, ohne die epikritische Bemerkung hinzuzufügen, dass in vorliegendem Falle das heute so in den Vordergrund geschobene ätiolo gische Moment der Lues fehlte. Weder in der Anamnese, noch durch die Untersuchung, noch durch die Obduktion liessen sich Handhaben einer vorausgegangenen Syphilis auffinden. Dagegen war Abusus potus nicht allein von dem Patienten zugestanden, sondern die Leber zeigte auch nachweisliche Spuren davon. Ebenso hatte anamnestisch eine häufig forcirte körperliche Muskelarbeit stattgefunden, ein Moment, das nach der Auffassung Scheele's bei der Entstehung von Aortenaneurysmen heutzutage meist viel zu sehr vernachlässigt wird.

Am Schlusse dieser Zeilen ist es mir eine angenehme Pflicht, meinem früheren Chef Herrn Geh. Rath Dr. Scheele in Wies baden für die liebenswürdige Berathung bei Abfassung der Arbeit meinen Dank auszusprechen. 